\title{
Commentary
}

\section{Counting apoptosis-why and how?}

The term apoptosis was coined 24 years ago to describe a morphological pattern of cell death recognised by several different authors in different scientific disciplines.' Despite the simplicity of the term, it is now clear that there are many different stimuli which can cause apoptosis, as well as a large number of distinct, but sometimes inter-related, genetically determined pathways which bring about its execution. Initial studies concentrated on biochemical events such as the disintegration of nuclear material into 180 base pair nucleosomal fragments. Other authors have identified changes in the cytoskeleton-for example, activation of tissue transglutaminase, ${ }^{2}$ and explored the use of these changes as a means of detecting and quantifying apoptosis. More recently, as apoptosis has enjoyed much wider exposure, there has been a plethora of new methods purported to identify apoptosis and permit accurate quantification. Indeed, some respected scientific journals have published statements claiming that one method or another is the only acceptable way of identifying and counting apoptosis. ${ }^{3}$ With increased knowledge of the complexity of the genetic pathways involved in regulating, and executing, apoptosis ${ }^{4}$ it is now apparent that many of the changes in nuclear and DNA structure, tissue cytoskeleton and antigen expression may be peculiar to certain cell types or apoptosis triggered in certain ways. Thus, a healthy degree of scepticism should be exercised when confronted with any novel assay that claims to be able both to define and identify this phenomenon.

Why then is there so much interest in the quantification of apoptosis in tissue sections? For many years apoptosis was regarded as a rather esoteric, quaint, but fundamentally unimportant phenomenon most often seen in vitro or perhaps in tumours. It was only after the rapidity of the process from initiation to removal of apoptotic fragments by phagocytosis was recognised, that it became clear that apparent low detectable levels of apoptosis could represent substantial cell loss. Thus, apoptosis moved from being the province of a select few to entering mainstream science, being implicated in everything from ontogeny of the lymphoid system to prion disease. The explosion of interest in apoptosis at many academic centres coincided with an increase in commercial research interest where pharmaceutical companies recognised the potential value of producing agents which control apoptosis. In addition, companies producing diagnostics realised that as many groups came into this new area there was potential to develop and market kits specifically designed to quantify the phenomenon. Indeed, such techniques have made apoptosis as an in vivo phenomenon accessible to many groups, but the down side perhaps has been a degree of confusion about how best to quantify apoptosis. A recent paper in this journal ${ }^{5}$ and a paper in this issue ${ }^{6}$ have addressed this question.

\section{Rationale of different methods for quantifying apoptosis} IN SITU TECHNIQUES

The in situ end labelling and in situ nick translation techniques rely on the presence of DNA strand breaks charac- teristic of the nuclear implosion and fragmentation seen in apoptosis. Thus, labelling with biotinylated nucleotides and subsequent immunodetection can be used to identity sensitively cells with strand breakage. It is clear that the ease of labelling nuclei varies and there are various proteinase and heat treatments available to enhance sensitivity. In addition to staining of tissue sections, the technique can be used to prepare cells for flow cytometry, thus permitting a very straightforward, quantifiable assessment of whether any particular nucleus is apoptotic or not. The identification of nuclei, designated positive or negative, tends to inspire confidence in the mind of the observer. There are, however, a number of possible limitations to this technique which should be considered. First, apoptosis is not the only situation in which sufficient DNA strand breaks may occur to allow detection. ${ }^{7}$ For example, necrosis, autolysis and (theoretically at least) DNA breaks which will be repaired subsequently have all been shown to label positively. Second, under some situations apoptosis may not result in the generation of nucleosomal fragments but in much larger fragments with presumably fewer detectable breaks. Third, for reasons which are not completely clear, not all apoptotic cells label.

\section{ANTIGENIC MARKERS OF APOPTOSIS}

The identification of protein markers permits ready detection by conventional immunohistochemistry, which facilitates rapid and confident assessment of apoptosis. It is important at this stage to remember that apoptosis is essentially a morphological state arrived at by a whole variety of different biochemical pathways. Some routes may result in the expression or loss of an antigen but there is no assurance that the same biochemical alterations occur in every cell. Transglutaminase expression in liver cell apoptosis, ${ }^{2}$ anti-apoptotic specific protein (ASP) antibody in lymphoma and reactive lymph nodes ${ }^{5}$ and enhanced expression of CD $11 \mathrm{~b} / \mathrm{CD} 18$ and CD $11 \mathrm{c} / \mathrm{CD} 18$ in apoptotic neutrophils ${ }^{8}$ have all been described. There is no reason to assume that these changes are anything other than cell lineage and apoptotic trigger specific. They may be extremely useful but only when properly validated in the specific cell lineage being studied.

\section{BACK TO MORPHOLOGY}

This leaves a third method-the recognition and quantification of apoptosis based on morphological appearances. Electron microscopy, while definitive, is impracticable and therefore apoptosis is counted on conventional haematoxylin and eosin or Feulgen stained sections. There are, of course, many objections to this, including doubts as to specificity, the problems of how to count a fragmented cell and the sensitivity of the technique. It is clear, however, that these criticisms are also applicable to the techniques described earlier and therefore are not of themselves reasons to dismiss the methodology.

\section{Conclusions}

Apoptosis is important even when present at low frequency in vivo because of its very short duration. It can be quanti- 
fied in an attempt to understand the balance of cell proliferation or death in regulated cell numbers. There are several different techniques available to quantify apoptosis, each based on some aspect of the biology of the process. However, there is a danger of losing sight of the morphological definition of apoptosis and, instead, measuring related or overlapping phenomena with mistaken confidence. The most universally applicable measuring technique is undoubtedly a fundamental histological assessment based on morphological criteria. Other techniques do indeed make assessment easier and are therefore to be welcomed. It is essential that whatever technique is adopted is developed for use in the appropriate system being studied. It is not sufficient to assume that because an in situ technique works in one tissue it will have exactly the same significance and connotations in another. Similarly, the use of an antibody in one system may not necessarily mean that the same antibody has a utility in another. Having said that, there is one final consideration that tends to be overlooked, the statistical validity of the sampling and collection of quantitative data. This is highlighted in the paper in this issue (pM273). In a previous study ${ }^{9}$ investigating the depletion of cells from lymph nodes following treatment with an antibody directed against CD4, a running mean was calculated. This gave an estimate of at least 5000 cells to be counted before statistical confidence could be applied to the results. Many papers choose a number of cells arbitrarily, varying from 100 to 300 , to be counted, and there is no guarantee that these numbers are sufficient for reasonable conclusions to be drawn, particularly if the rate of apoptosis is low. There is nothing inferior about a morphological assessment based on haematoxylin and eosin or Feulgen staining, but every study needs to be carefully validated statistically so that the numbers obtained by whatever technique are in fact credible.

University Department of Pathology,

DAVID J HARRISON

Medical School,

Teviot Place,

Edinburgh $E H 89 A G$

1 Kerr IF, Wyllie AH, Currie AR. Apoptosis: a basic biological phenomenon with wide ranging implications in tissue kinetics, $B r \mathcal{F}$ Cancer 1972;26: 239-57.

2 Fesus L, Thomazy V, Falus A. Induction and activation of tissue transglutaminase during programmed cell death. FEBS Lett 1987;224: $104-8$.

3 Shortman K, Scollay R. Death in the thymus. Nature 1994;372:44-5.

4 Bellamy COC, Malcomson RDG, Harrison DJ, Wyllie AH. Cell death in health and disease: the biology and regulation of apoptosis. Semin Cancer Biol 1995;6:3-16.

5 van de Schepop HAM, de Jong JS, van Diest PJ, Baak JPA. Counting of apoptotic cells: a methodological study in invasive breast cancer. 7 Clin Pathol: Mol Pathol 1996;49:M214-17.

6 Panchalingam S, Reynolds GM, Lammas DA, Rowlands D, Kumararatne DS. Simple method for pretreatment of tissue sections for the detection of apoptosis by in situ end labelling and in situ nick translation. 7 Clin Pathol: Mol Pathol 1996;49:M273-7.

7 Grasl-Kraupp B, Ruttkay-Nedecky B, Koudelka H, Bukowska K, Bursch W, Schulte-Hermann R. In situ detection of fragmented DNA (TUNEL assay) fails to discriminate among apoptosis, necrosis, and autolytic cell death: a cautionary note. Hepatology 1995;21:1465-8.

8 Dransfield I, Stocks SC, Haslett C. Regulation of cell adhesion molecule expression and function associated with neutrophil apoptosis. Blood 1995; 85:3264-73.

9 Howie SEM, Sommerfield AJ, Gray E, Harrison DJ. Peripheral T lymphocyte depletion by apoptosis after CD4 ligation in vivo: selective loss of CD44- and "activating" memory $\mathrm{T}$ cells. Clin Exp Immunol 1994;95:195-200. 